\title{
Fischer decomposition in generalized fractional ternary Clifford analysis*
}

\author{
P. Cerejeiras ${ }^{\ddagger}$, A. Fonseca ${ }^{\ddagger}$, M. Vajiac ${ }^{\dagger}$ and N. Vieira ${ }^{\ddagger}$ \\ † Chapman University, Department of Mathematics, \\ von Neumann Hall, Orange, CA 92866, U.S.A. \\ E-mail: mbvajiac@chapman.edu \\ ‡ CIDMA - Center for Research and Development in Mathematics and Applications \\ Department of Mathematics, University of Aveiro \\ Campus Universitário de Santiago, 3810-193 Aveiro, Portugal. \\ E-mail:pceres@ua.pt, aurineidefonseca@ufpi.edu.br,nloureirovieira@gmail.com
}

\begin{abstract}
This paper describes the generalized fractional Clifford analysis in the ternary setting. We will give a complete algebraic and analytic description of the spaces of monogenic functions in this sense, their analogous Fischer decomposition, concluding with a description of the basis of the space of fractional homogeneous monogenic polynomials that arise in this case and an explicit algorithm for the construction of this basis.

This paper is dedicated to professor Franciscus Sommen, on the occasion of his 60-th birthday. We wish him a long and happy life!
\end{abstract}

MSC 2010: 30G35; 26A33; 30A05; 31B05.

\section{Introduction}

In the classical physics and mathematics literature, the Dirac equation arises from the linearization of a relativistic second order wave equation by imposing an additional $S U(2)$-symmetry. While this describes an electron one would like to have other structures which impose an $S U(N)$-symmetry, for example supersymmetry, or the Calogero-Moser dynamical system of one-dimensional N-body problem of $\mathrm{N}$ equal particles with a harmonic potential. Another way of analyzing such structures can be found in $d$-fold factorizations; however, such a factorization is beyond the scope of classic partial derivatives and Clifford algebras.

One way to think about imposing a higher level symmetry is to combine the concepts of fractional derivatives and generalized Clifford Algebras [10]. But such a factorization runs into immediate problems from the point of view of quantum mechanics and, more mathematically speaking, from the point of view of a function theory, i.e. a theory of functions belonging to the Dirac operator that arises naturally.

To review, in the classic Clifford algebra setting, the construction of a monogenic function theory is based on the construction of a so-called Howe dual pair consisting of a Super-Lie algebra (usually osp (1|2)) and a Spinor space. This Super-Lie algebra $\mathfrak{o s p}(1 \mid 2)$ is then generated by three operators: the Dirac operator, the vector variable operator, and the so-called Euler operator or radial derivative; the latter operator arises as the anti-commutator between the Dirac operator and the vector variable operator and has as eigenspaces the space of homogeneous polynomials. But this construction immediately fails in the present case. The principal reason is that the choice of $\mathfrak{o s p}(1 \mid 2)$ is based on the preference of $S U(2)$ symmetries of the classic Clifford algebras

* The final version is published in Complex Analysis and Operator Theory, 11-No.5, (2017), 1077-1093. It as available via the website https://link.springer.com/article/10.1007/s11785-016-0631-7 
which are not preserved in the case of a generalized Clifford algebra (see [3]). In our case we will develop the specific symmetries and the different algebraic model that arises.

To summarize, in this paper we will follow a different path, approaching the problem through ways pioneered in hypercomplex analysis by F. Sommen, in the new context of generalized Clifford algebras. Following this road, instead of constructing a Super-Lie algebra associated with our structures we will construct a so-called Fischer pair base and introduce an inner product onto the space of homogeneous polynomials with values in the generalized Clifford algebra. It is very clear that this cannot be the same inner product as in the case of the classic Clifford algebra, but it can be produced by a clever introduction of a conjugation operator. While this allows for the construction of a Fischer decomposition we encounter an additional problem. Due to the lack of the $\mathfrak{o s p}(1 \mid 2)$-property, in particular the lack of a suitable Euler operator, we cannot use the standard ansatz for the monogenic projection, i.e. the projection of an arbitrary homogeneous polynomials into the space of monogenic homogeneous polynomials. To overcome this problem we are going to show that this projection can be recast as a linear system with coefficients in the generalized Clifford algebra. Although linear algebra with respect to Clifford-valued matrices is a difficult topic we can show that the resulting system is solvable in our case.

For the sake of simplicity and understanding of this paper we restrict ourselves to the case of $S U(3)$ symmetries, i.e. the ternary Clifford algebra. We will see that this algebra will provide a cubic factorization of the Laplacian and we will analyze the associated function theory. In the last part of the paper we will use a computer algebra system to compute the coefficients of the monogenic homogeneous polynomials that form the basis of the space of fractional homogeneous monogenic polynomials that arise in this case. For the actual calculation of the monogenic basis polynomials we provide a MATLAB program which can be easily adapted to larger calculations as well.

For the definition and some applications of generalized Clifford algebras, including the case of $d=3$, we point the reader to the works of Traubenberg and others $[11,17,7]$, though these papers do not have the same scope in the context of $N$-fold factorizations of the Laplacian.

\section{Preliminaries}

\subsection{Generalized Clifford algebras in dimension 3}

It is well known that the treatment of the two-dimensional vector spaces $\mathbb{R}^{2}$ in terms of complex numbers has the advantage of containing an intrinsic multiplicative structure. Appropriate higher-dimensional associative analogues of the complex numbers are the real Clifford algebras. For details about Clifford algebras and basic concepts of the associated function theory we refer the interested reader to $[1,2,9]$. However, to obtain a Dirac operator $D$ such that $D^{3}=\Delta$ a real Clifford algebra is not enough and we need to define a so-called generalized Clifford algebra. In what follows we give a detailed description of the "ternary" Clifford algebra that we use in this case. Let $\left\{e_{1}, \cdots, e_{d}\right\}$ be the standard basis of the Euclidean vector space in $\mathbb{C}^{d}$, i.e. the standard basis for the Euclidean vector space in $\mathbb{R}^{d}$, which is then complexified. The associated ternary Clifford algebra $\mathrm{Cl}_{d}^{1 / 3}$ is the free algebra generated by $\mathbb{C}^{d}$ subject to the multiplication rule:

$$
e_{i}^{3}=1, \quad e_{i} e_{j}=\omega e_{j} e_{i}, \text { for } 1 \leq i<j \leq d,
$$

where $\omega=e^{i 2 \pi / 3}$. These multiplication rules are a consequence of the following relation:

$$
\left[e_{i}, e_{j}, e_{k}\right]:=e_{i} e_{j} e_{k}+e_{i} e_{k} e_{j}+e_{j} e_{i} e_{k}+e_{j} e_{k} e_{i}+e_{k} e_{i} e_{j}+e_{k} e_{j} e_{i}=6 \delta_{i j k},
$$

for all $i, j, k=1, \ldots, d$, where the form $\left[e_{i}, e_{j}, e_{k}\right]$ defined above is an extension of the anti-commutator relation in the ternary setting. A vector space basis for $\mathcal{C}_{d}^{1 / 3}$ is given by the set of all ordered products:

$$
e^{\nu}:=e_{1}^{\nu_{1}} \cdots e_{d}^{\nu_{d}}
$$

where $\boldsymbol{\nu}=\left(\nu_{1}, \cdots, \nu_{d}\right)$ is an ordered $d$-tuple with $\nu_{j}=0,1,2$. We note that, using (1) $e_{i} e_{j}=\omega e_{j} e_{i}$ implies $e_{j} e_{i}=\omega^{2} e_{i} e_{j}$, for all $1 \leq i<j \leq d$. From these relations one obtains the following commutator relation:

$$
e_{i}^{\nu_{i}} e_{j}^{\mu_{j}}=\omega^{\nu_{i} \mu_{j}} e_{j}^{\mu_{j}} e_{i}^{\nu_{i}}, \quad e_{j}^{\nu_{j}} e_{i}^{\mu_{i}}=\omega^{2 \nu_{j} \mu_{i}} e_{i}^{\mu_{i}} e_{j}^{\nu_{j}}, \quad 1 \leq i<j \leq d,
$$


which leads to the multiplication rule between the elements of the basis (3) as:

$$
e^{\nu} e^{\boldsymbol{\mu}}=\omega^{\boldsymbol{\nu} * \boldsymbol{\mu}} e^{\boldsymbol{\nu}+\boldsymbol{\mu}}
$$

with

$$
\boldsymbol{\nu} * \boldsymbol{\mu}:=2\left(\nu_{d}+\nu_{d-1}+\cdots+\nu_{2}\right) \mu_{1}+2\left(\nu_{d}+\nu_{d-1}+\cdots+\nu_{3}\right) \mu_{2}+\cdots+2 \nu_{d} \mu_{d-1}=2 \sum_{j=1}^{d-1} \sum_{s=j+1}^{d} \nu_{s} \mu_{j} .
$$

Here we see that, due to (1), these products have to be understood as elements of a modulus 3 class, and one obtains relations of the following type:

$$
e_{j}^{1} e_{j}^{2}=e_{j}^{3}=e_{j}^{0}=1, \quad \text { or } \quad e_{j}^{2} e_{j}^{2}=e_{j}^{4}=e_{j}
$$

Each $a \in \mathcal{C} \ell_{d}^{1 / 3}$ can be written in the form $a=\sum_{\nu} a_{\boldsymbol{\nu}} e^{\boldsymbol{\nu}}$, with $a_{\boldsymbol{\nu}} \in \mathbb{C}$. Therefore, the ternary Clifford algebra $\mathrm{Cl}_{d}^{1 / 3}$ has the form:

$$
\mathcal{C}_{d}^{1 / 3}=\left\{w=\sum_{\nu} w_{\nu} e^{\nu}, w_{\nu} \in \mathbb{C}, \boldsymbol{\nu}=\left(\nu_{1}, \cdots, \nu_{d}\right), \nu_{j}=0,1,2\right\}
$$

where we recall the complex scalars $z \in \mathbb{C}$ commute with the basis elements, i.e., $z e^{\boldsymbol{\nu}}=e^{\boldsymbol{\nu}} z$. It can easily be proved that for a vector $w=w_{1} e_{1}+\cdots+w_{d} e_{d}$ we have $w^{3}=w_{1}^{3}+\cdots+w_{d}^{3} \in \mathbb{C}$. Therefore, a vector in $\mathcal{C} \ell_{d}^{1 / 3}$ is invertible if and only if $w^{3} \neq 0$. The conjugation in this ternary Clifford algebra $\mathcal{C l}_{d}^{1 / 3}$ is, by definition, the involutory automorphism ${ }^{-}: \mathcal{C}_{d}^{1 / 3} \rightarrow \mathcal{C} \ell_{d}^{1 / 3}$ given by:

$$
w \mapsto \bar{w}=\sum_{\nu} \bar{w}_{\nu} \overline{e^{\nu}}
$$

where $\bar{w}_{\boldsymbol{\nu}}$ denotes the usual complex conjugation and its action on the basis elements is defined by:

$$
\overline{u w}=\bar{w} \bar{u}, \quad u, w \in \mathcal{C} \ell_{d}^{1 / 3}
$$

together with

$$
\overline{e_{j}^{\nu_{j}}}=e_{j}^{3-\nu_{j}}, \quad \nu_{j}=0,1,2, j=1, \cdots, d
$$

Therefore, one obtains:

$$
\overline{e^{\boldsymbol{\nu}}}=\overline{e_{1}^{\nu_{1}} \cdots e_{d}^{\nu_{d}}}:=e_{d}^{3-\nu_{d}} \ldots e_{1}^{3-\nu_{1}}
$$

and this element can be expressed in terms of the chosen basis elements (3) as:

$$
\overline{e^{\boldsymbol{\nu}}}=e_{d}^{3-\nu_{d}} \ldots e_{1}^{3-\nu_{1}}=\omega^{\nu^{*}} e^{3-\nu}
$$

where $3-\boldsymbol{\nu}:=\left(3-\nu_{1}, \cdots, 3-\nu_{d}\right)$ and:

$$
\nu^{*}:=2 \sum_{j=1}^{d-1} \sum_{s=j+1}^{d}\left(3-\nu_{j}\right)\left(3-\nu_{s}\right)
$$

where we remind the reader that these products are to be understood as elements of a modulus 3 class. For more details on these generalized Clifford algebras the reader is invited to consult Fleury, Traubenberg and Jagannathan's work in $[7,11,17]$.

In what follows, let $\Omega$ denotes a domain of $\mathbb{C}^{d}$ with the usual topologies. We shall consider functions $f: \Omega \subset \mathbb{C}^{d} \rightarrow \mathcal{C}_{d}^{1 / 3}$, where,$x=\left(x_{1}, \ldots, x_{d}\right) \in \Omega \mapsto f(x)=\sum_{\nu} f_{\nu}(x) e^{\nu}$, with $f_{\nu}: \Omega \subset \mathbb{C}^{d} \rightarrow \mathbb{C}^{\text {. Properties }}$ will be ascribed to $f$ if and only if all of its components $f_{\boldsymbol{\nu}}$ satisfy it. For example, $f$ is $C^{1}$ if and only if all $f_{\boldsymbol{\nu}}$ are $C^{1}$. 


\subsection{Generalized fractional derivatives}

In this section we recall some basic facts about generalized fractional calculus (for more details we refer [14]). We start with the following definition of generalized differentiation and integration operators.

Definition $2.1([\mathbf{8}, \mathbf{1 4}])$ Let the function

$$
\varphi(\lambda)=\sum_{k=0}^{\infty} \varphi_{k} \lambda^{k}
$$

be an entire function with order $\rho>0$ and degree $\sigma>0$. We define the linear operator $D_{\varphi}$, which acts on powers of $z^{n}$ as

$$
D_{\varphi} z^{0}:=0, \quad D_{\varphi} z^{n}:=\frac{\varphi_{n-1}}{\varphi_{n}} z^{n-1}, \quad n=1,2, \cdots
$$

We call $D_{\varphi}$ the fractional derivative associated to $\varphi$. The operation

$$
f(z)=\sum_{k=0}^{\infty} a_{k} z^{k} \stackrel{D_{\varphi}}{\longrightarrow} D_{\varphi} f(z)=\sum_{k=1}^{\infty} a_{k} \frac{\varphi_{k-1}}{\varphi_{k}} z^{k-1}
$$

is said to be the Gelfond-Leontiev $(G-L)$ operator of generalized differentiation with respect to the function $\varphi$, and the corresponding $G-L$ integration operator is:

$$
I_{\varphi} f(z)=\sum_{k=0}^{\infty} a_{k} \frac{\varphi_{k+1}}{\varphi_{k}} z^{k+1} .
$$

From the conditions required for $\varphi$ we have $\limsup _{k \rightarrow \infty} k^{1 / \rho} \sqrt[k]{\left|\varphi_{k}\right|}=(\sigma \rho \mathrm{e})^{1 / \rho}$, and thus $(\operatorname{see}[15,13])$

$$
\lim \sup _{k \rightarrow \infty} \sqrt[k]{\left|\frac{\varphi_{k-1}}{\varphi_{k}}\right|}=1
$$

Therefore, by the Cauchy-Hadamard formula, both series, (12) and (13), inherit the radius of convergence $R>0$ of $f$.

Example 2.2 Let $\varphi(\lambda)$ be the Mittag-Leffler function of the form

$$
\varphi(\lambda)=E_{\frac{1}{\rho}, \mu}(\lambda)=\sum_{k=0}^{\infty} \frac{\lambda^{k}}{\Gamma\left(\mu+\frac{k}{\rho}\right)}, \quad \rho>0, \quad \mu \in \mathbb{C},
$$

with $\operatorname{Re}(\mu)>0$. Then $\varphi_{k}(\lambda)=\frac{1}{\Gamma\left(\mu+\frac{k}{\rho}\right)}$ and operators (12), (13) turn into the so-called Dzrbashjan-GelfondLeontiev (D-G-L) operators of differentiation and integration:

$$
D_{\rho, \mu} f(z)=\sum_{k=1}^{\infty} a_{k} \frac{\Gamma\left(\mu+\frac{k}{\rho}\right)}{\Gamma\left(\mu+\frac{k-1}{\rho}\right)} z^{k-1}, \quad I_{\rho, \mu} f(z)=\sum_{k=0}^{\infty} a_{k} \frac{\Gamma\left(\mu+\frac{k}{\rho}\right)}{\Gamma\left(\mu+\frac{k+1}{\rho}\right)} z^{k+1},
$$

studied in [4, 5, 14].

In $[14,16]$ the author studied the connections between the D-G-L operators (14) and the so-called Erdélyi-Kober (E-K) fractional integrals and derivatives. In [14], the author presented transmutation operators relating the Riemann-Liouville (R-L) fractional integrals $R^{\frac{1}{\rho}}$ with the D-G-L generalized integrations $I_{\rho, 1}$, and $I_{\rho, \mu}$, which where given in terms of E-K operators. One can remark that, when more exotic derivatives are used, like Riemann-Liouville, for example, then relation (11) is affected by a "ground state" function $1[z]$, which is in general non-analytic, satisfying:

$$
D_{\varphi} z^{n}=(\text { up to const. depending on } n) z^{n-1} 1[z], n=0,1,2, \ldots
$$

This in turns, requires an appropriate branch cut in the analytic domain. However, these kind of derivatives are out of the scope of the present paper. We remark, nevertheless, that under certain regularity conditions on a bounded real interval $[0, b]$ these derivatives do coincide and satisfy the semigroup property $D^{\alpha} D^{\beta} u=D^{\alpha+\beta} u$ 
(see [6]). The above statements lead us to consider the ternary Dirac operator $D=\sum_{j=1}^{d} e_{j} D_{j}^{\alpha}$, where $D_{j}^{\alpha}$ represents the G-L generalized derivative (12) associated with the Mittag-Leffler function

$$
E_{\alpha, 1}(z)=\sum_{k=0}^{\infty} \frac{z^{k}}{\Gamma(1+k \alpha)}, \quad 0<\alpha<1,
$$

with respect to the $j$-coordinate. We emphasize that, as we use G-L derivatives associated to $E_{\alpha, 1}$, the radius of convergence of the original series will remain unchanged under differentiation or integration procedures. Giving a starlike open domain $\Omega$ in $\mathbb{C}^{d}$ and a (scalar-valued) function $u: \Omega \subset \mathbb{C}^{d} \rightarrow \mathbb{C}$, we have:

$$
\Delta^{3 \alpha / 2} u:=D^{3} u=\left(D_{1}^{\alpha}\right)^{3} u+\cdots+\left(D_{d}^{\alpha}\right)^{3} u, \quad \text { in } \Omega .
$$

Analogous to the Euclidean case a $C \ell_{d}^{1 / 3}$-valued function $u$ is called ternary left-monogenic if it satisfies $D u=0$ on $\Omega$ (resp. ternary right-monogenic if it satisfies $u D=0$ on $\Omega$ ). As can be seen from the above exposition the most common fractional derivatives arise as special cases in our studies. We start with the discussion of one of the most important tools in Clifford analysis, the Fischer decomposition.

\section{Fractional Fischer decomposition}

The aim of this section is to provide the basic tools for a function theory for the ternary Dirac operator defined via generalized Gelfond-Leontiev differentiation operators. As we mentioned before, the standard approach to the establishment of a function theory in higher dimensions is the construction of the analogues to the Euler and Gamma operators and the establishment of the corresponding Sommen-Weyl relations. However, in our case we cannot follow this path directly, since our generalized Clifford algebra does not have the necessary structure, therefore we will follow the more classic approach via the Fischer inner product. As we mentioned before, we consider the ternary Dirac operator $D=\sum_{j=1}^{d} e_{j} D_{j}^{\alpha}$, where $D_{j}^{\alpha}$ represents the fractional derivative associated to the Mittag-Leffler function $E_{\alpha, 1}$ with respect to the $j$-coordinate $(0<\alpha<1)$. Therefore, for $x_{l} \in \mathbb{C}$ we obtain (see (11)):

$$
D_{j}^{\alpha} x_{l}^{0}=0, \quad D_{j}^{\alpha} x_{l}=\frac{\varphi_{0}}{\varphi_{1}} \delta_{j, l}:=\varphi(1,0) \delta_{j, l},
$$

for all $j, l=1, \ldots, d$, where

$$
\varphi(k, l)=\frac{\Gamma(1+k \alpha)}{\Gamma(1+l \alpha)}, \quad k, l=0,1,2, \ldots,
$$

which imply that: $D_{j}^{\alpha} x_{l}=\Gamma(1+\alpha) \delta_{j, l}$. We will first analyze how the differential operators act on the variables $x_{i}$ :

$$
\begin{aligned}
\left(D_{j}^{\alpha}\right)^{k} x_{j}^{l} & =\varphi(l, l-1)\left(D_{j}^{\alpha}\right)^{k-1} x_{j}^{l-1} \\
& =\varphi(l, l-1) \varphi(l-1, l-2)\left(D_{j}^{\alpha}\right)^{k-2} x_{j}^{l-2} \\
& \vdots \\
& =\varphi(l, l-1) \varphi(l-1, l-2) \cdots \varphi(l-k+1, l-k) x_{j}^{l-k} .
\end{aligned}
$$

Therefore at $x_{j}=0$ we obtain:

$$
\left.\left(D_{j}^{\alpha}\right)^{k} x_{j}^{l}\right|_{x_{j}=0}= \begin{cases}0, & \text { if } k \neq l \\ \varphi(l, l-1) \cdots \varphi(1,0) & \text { if } k=l\end{cases}
$$

and we write $\varphi(l, l-1) \cdots \varphi(1,0)=\Gamma(1+l \alpha):=\Phi_{l}$. We will begin by analyzing the Fischer decomposition on the right module of polynomials, more specifically, on their building blocks, homogeneous polynomials of degree $n$. In fact, any homogeneous polynomial with coefficients in our algebra can be written as:

$$
P_{n}(\boldsymbol{X})=\sum_{\boldsymbol{l} \in \mathbb{N}_{0}^{d}:|\boldsymbol{l}|=n} \boldsymbol{X}^{\boldsymbol{l}} a_{\boldsymbol{l}}, \quad a_{\boldsymbol{l}} \in \mathcal{C} \ell_{d}^{1 / 3},
$$

with $\boldsymbol{l} \in \mathbb{N}_{0}^{d}, n=|\boldsymbol{l}|=l_{1}+\ldots+l_{d}$ denoting the degree of the polynomial, $\boldsymbol{X}^{\boldsymbol{l}}=x_{1}^{l_{1}} \cdots x_{d}^{l_{d}}$ and $a_{\boldsymbol{l}} \in \mathcal{C} \ell_{d}^{1 / 3}$ has the form $a_{\boldsymbol{l}}=\sum_{\boldsymbol{\nu}} a_{\boldsymbol{l}, \boldsymbol{\nu}} e^{\boldsymbol{\nu}}$. The Fischer inner product of two fractional homogeneous polynomials $P$ and $Q$ of degree $n$ is given by

$$
\langle P, Q\rangle_{n}=\left.\operatorname{Sc}[\bar{P}(\boldsymbol{\partial}) Q(\boldsymbol{X})]\right|_{x_{1}=\cdots=x_{d}=0},
$$


where $\bar{P}(\boldsymbol{\partial})$ is a differential operator obtained by replacing in the polynomial $\bar{P}$ each variable by its corresponding fractional derivative and Sc represents the scalar part of this product, that is, the coefficient of $e^{(0, \cdots, 0)}$. It is easy to check that (21) defines an inner product and we leave the details to the reader.

For fractional homogeneous polynomials of degree $n, P_{n}(\boldsymbol{X})=\sum_{\boldsymbol{l} \in \mathbb{N}_{0}^{d}:|l|=n} \boldsymbol{X}^{\boldsymbol{l}} a_{\boldsymbol{l}}$ and $Q_{n}(\boldsymbol{X})=\sum_{\boldsymbol{k} \in \mathbb{N}_{0}^{d}:|\boldsymbol{k}|=n} \boldsymbol{X}^{\boldsymbol{k}} b_{\boldsymbol{k}}$, we obtain

$$
\begin{aligned}
\langle P, Q\rangle_{n} & =\sum_{|\boldsymbol{l}|=n|\boldsymbol{k}|=n} \bar{a}_{\boldsymbol{l}}\left(\left.\left(D_{d}^{\alpha}\right)^{l_{d}} \cdots\left(D_{1}^{\alpha}\right)^{l_{1}} x_{1}^{k_{1}} \cdots x_{d}^{k_{d}}\right|_{x_{1}=\cdots=x_{d}=0}\right) b_{\boldsymbol{k}} \\
& =\sum_{|\boldsymbol{l}|=n} \overline{a_{\boldsymbol{l}}}\left(\prod_{l_{j} \neq 0} \Phi_{l_{j}}\right) b_{\boldsymbol{l}}:=\sum_{|\boldsymbol{l}|=n} \overline{a_{\boldsymbol{l}}} b_{\boldsymbol{l}} \Phi_{\boldsymbol{l}},
\end{aligned}
$$

where $\Phi_{l}=\prod_{j=1, l_{j} \neq 0}^{d} \Phi_{l_{j}}$. From (21) we immediately obtain that for any polynomial $P_{n-1}$ of homogeneity $n-1$ and any polynomial $Q_{n}$ of homogeneity $n$ the following holds:

$$
\left\langle X P_{n-1}, Q_{n}\right\rangle_{n}=\left\langle P_{n-1}, D Q_{n}\right\rangle_{n-1},
$$

where $X=x_{1} e_{1}+\cdots+x_{d} e_{d}$, and this fact allows us to prove the following result:

Theorem 3.1 For each $n \in \mathbb{N}_{0}$ we have $\Pi_{n}=\mathcal{M}_{n}+X \Pi_{n-1}$, where $\Pi_{n}$ denotes the space of fractional homogeneous polynomials of degree $n$ and $\mathcal{M}_{n}$ denotes the space of fractional monogenic homogeneous polynomials of degree $n$. Moreover, the subspaces $\mathcal{M}_{n}$ and $X \Pi_{n-1}$ are orthogonal with respect to the Fischer inner product (21).

Proof: Since $\Pi_{n}=X \Pi_{n-1}+\left(X \Pi_{n-1}\right)^{\perp}$, it suffices to prove that $\left(X \Pi_{n-1}\right)^{\perp}=\mathcal{M}_{n}$. Assume that $P_{n} \in \Pi_{n}$ is in $\left(X \Pi_{n-1}\right)^{\perp}$. Then, we have $\left\langle X P_{n-1}, P_{n}\right\rangle_{n}=0$, for all $P_{n-1} \in \Pi_{n-1}$. From (22) we get $\left\langle P_{n-1}, D P_{n}\right\rangle_{n-1}=0$, for all $P_{n-1} \in \Pi_{n-1}$. Hence, we obtain that $D P_{n}=0$, that is $P_{n} \in \mathcal{M}_{n}$. This means that $\left(X \Pi_{n-1}\right)^{\perp} \subset \mathcal{M}_{n}$. Conversely, take $P_{n} \in \mathcal{M}_{n}$. Then, for every $P_{n-1} \in \Pi_{n-1}$ we have that

$$
\left\langle X P_{n-1}, P_{n}\right\rangle_{n}=\left\langle P_{n-1}, D P_{n}\right\rangle_{n-1}=\left\langle P_{n-1}, 0\right\rangle_{n-1}=0,
$$

from which it follows that $\mathcal{M}_{n} \subset\left(X \Pi_{n-1}\right)^{\perp}$. Therefore $\mathcal{M}_{n}=\left(X \Pi_{n-1}\right)^{\perp}$.

In consequence, we obtain the fractional Fischer decomposition with respect to the fractional Dirac operator $D$. However, in order to obtain further decompositions of the space $\Pi_{n}$ we need first to study the commutator relations between the fractional derivatives and variables acting on fractional powers:

$$
\begin{aligned}
{\left[D_{i}^{\alpha}, x_{j}\right] x_{r}^{l} } & =\left(D_{i}^{\alpha} x_{j}-x_{j} D_{i}^{\alpha}\right) x_{r}^{l} \\
& = \begin{cases}0, & \text { if } i \neq j \\
\varphi(1,0) x_{r}^{l}, & \text { if } i=j \wedge i \neq r \\
(\varphi(l+1, l)-\varphi(l, l-1)) x_{r}^{l}, & \text { if } i=j=r\end{cases}
\end{aligned}
$$

with $l \in \mathbb{N}, i, j, r=1, \ldots, d$.

Example 3.2 For the case where $\alpha=2 / 3$ we have $\varphi(k, l)=\frac{\Gamma\left(1+\frac{2 k}{3}\right)}{\Gamma\left(1+\frac{2 l}{3}\right)}$. Therefore,

$$
\left[D_{i}^{2 / 3}, x_{j}\right] x_{r}^{l}=\left\{\begin{array}{ll}
0, & \text { if } i \neq j \\
\Gamma(5 / 3) x_{r}^{l}, & \text { if } i=j \wedge i \neq r \\
\left(\frac{\Gamma\left(1+\frac{2(l+1)}{3}\right)}{\Gamma\left(1+\frac{2 l}{3}\right)}-\frac{\Gamma\left(1+\frac{2 l}{3}\right)}{\Gamma\left(1+\frac{2(l-1)}{3}\right)}\right) x_{r}^{l}, & \text { if } i=j=r
\end{array} .\right.
$$

Here we remind the reader that, under certain regularity conditions, the D-G-L derivatives enjoy the semi-group property. Hence, the choice of parameter $\alpha=\frac{2}{3}$ ensures that (16) relates to the standard Laplace operator, that is,

$$
D^{3} u=\left(D_{1}^{2 / 3}\right)^{3} u+\cdots+\left(D_{d}^{2 / 3}\right)^{3} u:=\Delta u .
$$

Hence, in what follows we shall consider $\alpha=\frac{2}{3}$. 
Theorem 3.3 Let $P_{n}$ be a fractional homogeneous polynomial of degree $n$. Then we have:

$$
P_{n}=M_{n}+X M_{n-1}+X^{2} M_{n-2}+\ldots+X^{n} M_{0},
$$

where each $M_{j}$ denotes the fractional monogenic polynomial of degree $j$. More specifically,

$$
M_{0} \in \Pi_{0}, \quad \text { and } \quad M_{n} \in\left\{u \in \Pi_{n}: \quad D u=0\right\} .
$$

Corollary 3.4 Let $H_{n}$ be a fractional homogeneous harmonic polynomial of degree $n$, id est $0=\Delta H_{n}:=D^{3} H_{n}$. Then $H_{n}$ has the form:

$$
H_{n}=M_{n}+X M_{n-1}+X^{2} M_{n-2}
$$

The spaces represented in (24) are orthogonal to each other with respect to the Fischer inner product (21). Moreover, the above decomposition can be represented in form of an infinite triangle:

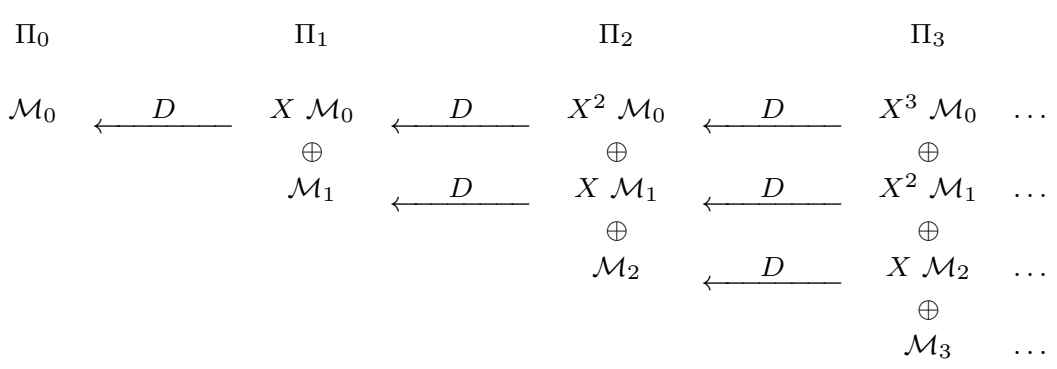

All the spaces in the above diagrams are right modules, the Dirac operator shifts all spaces in the same row to the left while the multiplication by $X$ shifts them to the right, and both of these actions establish isomorphisms between the respective modules. From Theorem 3.3 we can derive the following direct extension to the fractional case of the Almansi decomposition:

Theorem 3.5 For any fractional polyharmonic polynomial $P_{n}$ of degree $n \in \mathbb{N}_{0}$ in a starlike domain $\Omega$ in $\mathbb{C}^{d}$ with respect to 0 , i.e.,

$$
D^{3 n} P_{n}=0, \text { in } \Omega
$$

there exist uniquely fractional harmonic functions $P_{0}, P_{1}, \ldots, P_{n-1}$ such that

$$
P_{n}=P_{0}+X^{3} P_{1}+\ldots+X^{3(n-1)} P_{n-1} \text { in } \Omega \text {. }
$$

\subsection{Explicit formulae}

The aim of this subsection is to give an explicit algorithm for the construction of the projection $\pi_{\mathcal{M}}\left(P_{n}\right)$ of a given fractional homogeneous polynomial $P_{n}$ into the space of fractional homogeneous monogenic polynomials $\mathcal{M}_{n}$. In order to reach our goal, we start by looking at the dimension of the space of fractional homogeneous monogenic polynomials of degree $n$. From the Fischer decomposition (24) we obtain:

$$
\operatorname{dim}\left(\mathcal{M}_{n}\right)=\operatorname{dim}\left(\Pi_{n}\right)-\operatorname{dim}\left(\Pi_{n-1}\right)
$$

with the dimension of the space of fractional homogeneous polynomials of degree $n$ given by

$$
\operatorname{dim}\left(\Pi_{n}\right)=\frac{(n+d-1) !}{n !(d-1) !}=\left(\begin{array}{c}
n+d-1 \\
d-1
\end{array}\right) .
$$

This leads to the following theorem:

Theorem 3.6 The space of fractional homogeneous monogenic polynomials of degree $n$ has dimension

$$
\operatorname{dim}\left(\mathcal{M}_{n}\right)=\frac{(n+d-1) !}{n !(d-1) !}-\frac{(n-1+d-1) !}{(n-1) !(d-1) !}=\frac{(n+d-2) !}{n !(d-2) !}=\left(\begin{array}{c}
n+d-2 \\
d-2
\end{array}\right)
$$


In the classical setting (see $[2,12,18]$ ) one usually considers the following scheme for the monogenic projection:

$$
r=a_{0} P_{n}+a_{1} X D P_{n}+a_{2} X^{2} D^{2} P_{n}+\ldots+a_{n} X^{n} D^{n} P_{n},
$$

with $a_{j} \in \mathbb{C}, j=0, \ldots, l$, and $a_{0}=1$. This approach, unfortunately, does not work in our case; this fact is, of course, due to the lack of the $\mathfrak{o s p}(1 \mid 2)$ property. Using the Fischer decomposition and the explicit knowledge of the dimensions of the spaces (see Theorem 3.6) we can use a more direct approach to determine the fractional homogeneous monogenic polynomial. As we have seen before, any homogeneous polynomial of degree $n=|\boldsymbol{l}|$ with coefficients in $\mathcal{C} \ell_{d}^{1 / 3}$ can be written as

$$
P_{n}(\boldsymbol{X})=\sum_{\boldsymbol{l} \in \mathbb{N}_{0}^{d}:|\boldsymbol{l}|=n} \boldsymbol{X}^{\boldsymbol{l}} a_{\boldsymbol{l}}, \quad a_{\boldsymbol{l}} \in \mathcal{C l}_{d}^{1 / 3}
$$

with $n=|\boldsymbol{l}|=l_{1}+\ldots+l_{d}$ denoting the degree of the polynomial. We now check under which conditions we have $D P_{n}=0$, i.e.,

$$
\begin{aligned}
0= & D\left(\sum_{\boldsymbol{l} \in \mathbb{N}_{0}^{d}:|\boldsymbol{l}|=n} \boldsymbol{X}^{\boldsymbol{l}} a_{\boldsymbol{l}}\right)=\sum_{\boldsymbol{l} \in \mathbb{N}_{0}^{d}:|\boldsymbol{l}|=n}\left(D \boldsymbol{X}^{\boldsymbol{l}}\right) a_{\boldsymbol{l}} \\
= & \sum_{\boldsymbol{l} \in \mathbb{N}_{0}^{d}:|\boldsymbol{l}|=n}\left(\sum_{j=1}^{d} e_{j} D_{j}^{2 / 3} x_{1}^{l_{1}} \cdots x_{d}^{l_{d}}\right) a_{\boldsymbol{l}} \\
= & e_{1} \varphi(n, n-1) x_{1}^{n-1} a_{(n, 0, \cdots, 0)} \\
& +\left[e_{1} \varphi(n-1, n-2) x_{1}^{n-2} x_{2}+e_{2} \varphi(1,0) x_{1}^{n-1}\right] a_{(n-1,1,0, \cdots, 0)} \\
& +\cdots+\left[e_{1} \varphi(n-1, n-2) x_{1}^{n-2} x_{d}+e_{d} \varphi(1,0) x_{1}^{n-1}\right] a_{(n-1,0, \cdots, 0,1)} \\
& +\left[e_{1} \varphi(n-2, n-3) x_{1}^{n-3} x_{2}^{2}+e_{2} \varphi(2,1) x_{1}^{n-2} x_{2}\right] a_{(n-2,2,0, \cdots, 0)} \\
& +\left[e_{1} \varphi(n-2, n-3) x_{1}^{n-3} x_{2} x_{3}+e_{2} \varphi(1,0) x_{1}^{n-2} x_{3}+e_{3} \varphi(1,0) x_{1}^{n-2} x_{2}\right] a_{(n-2,1,1,0 \cdots, 0)} \\
& +\cdots+\left[e_{1} \varphi(n-2, n-3) x_{1}^{n-3} x_{2}^{2}+e_{d} \varphi(2,1) x_{1}^{n-2} x_{d}\right] a_{(n-2,0, \cdots, 0,2)} \\
& +\cdots+e_{d} \varphi(n, n-1) x_{d}^{n-1} a_{(0, \cdots, 0, n)}
\end{aligned}
$$

The last equality leads to the following theorem:

Theorem 3.7 Equation (26) is equivalent to the following linear system:

$$
M A=0
$$

where $A=\left[a_{\left(l_{1}, \ldots, l_{d}\right)}\right]_{\operatorname{dim}\left(\Pi_{n}\right) \times 1}, 0=[0]_{\operatorname{dim}\left(\Pi_{n-1}\right) \times 1}$ are vectors, and $M$ is the matrix

$$
M=\left[M_{\left(k_{1}, \ldots, k_{d}\right),\left(l_{1}, \ldots, l_{d}\right)}\right]_{\operatorname{dim}\left(\Pi_{n-1}\right) \times \operatorname{dim}\left(\Pi_{n}\right)},
$$

with entrances given by

$$
M_{\left(k_{1}, \ldots, k_{d}\right),\left(l_{1}, \ldots, l_{d}\right)}=\left\{\begin{array}{cc}
e_{i} \varphi\left(l_{i}, k_{i}\right), & k_{i}=l_{i}-1 \wedge k_{j}=l_{j} \forall i \neq j \\
0, & \text { others cases }
\end{array} .\right.
$$

Let us now indicated a possible ordering for the rows of system (27). In order to proceed, let us consider the following ordered set:

$$
L=\left\{\underline{L}^{i}=\left(l_{1}^{i}, \ldots, l_{d}^{i}\right): \quad\left|\underline{L}^{i}\right|=n=l_{1}^{i}+\ldots+l_{d}^{i}, \quad i=1,2 \ldots, \operatorname{dim}\left(\Pi_{n}\right)\right\},
$$

where the relation order is given by

$$
\underline{L}^{i}>\underline{L}^{i+1} \Leftrightarrow\left(l_{1}^{i}, \ldots, l_{d}^{i}\right)>\left(l_{1}^{i+1}, \ldots, l_{d}^{i+1}\right) \Leftrightarrow l_{i}^{i} l_{2}^{i} \ldots l_{d}^{i}>l_{1}^{i+1} l_{2}^{i+1} \ldots l_{d}^{i+1}
$$

with

$$
l_{1}^{k} l_{2}^{k} \ldots l_{d}^{k}:=l_{1}^{k} \times 10^{d-1}+l_{2}^{k} \times 10^{d-2}+\ldots+l_{d}^{k} \times 10^{0} .
$$

Applying this ordering we get the following corollary. 
Corollary 3.8 The matrix $M$ has the following structure:

$$
M=\left(\begin{array}{ll}
M_{1} & M_{2}
\end{array}\right)
$$

where the sub-matrix $M_{1}=\left[m_{i j}^{1}\right]_{\operatorname{dim}\left(\Pi_{n-1}\right) \times \operatorname{dim}\left(\Pi_{n-1}\right)}$ is an upper triangular matrix with entrances given by:

$$
\begin{aligned}
& M_{1}=\left(\begin{array}{ccccc}
e_{1} \varphi(n, n-1) & e_{2} \varphi(1,0) & e_{3} \varphi(1,0) & e_{4} \varphi(1,0) & e_{5} \varphi(1,0) \\
0 & e_{1} \varphi(n-1, n-2) & 0 & e_{2} \varphi(2,1) & e_{3} \varphi(1,0) \\
0 & 0 & e_{1} \varphi(n-1, n-2) & 0 & e_{2} \varphi(1,0) \\
\vdots & \vdots & \vdots & \ddots & \ddots \\
0 & 0 & 0 & 0 & 0
\end{array}\right. \\
& \left.\begin{array}{cccccc}
\cdots & e_{d} \varphi(1,0) & 0 & 0 & \cdots & 0 \\
\cdots & e_{d-2} \varphi(1,0) & e_{d-1} \varphi(1,0) & e_{d} \varphi(1,0) & \cdots & 0 \\
\cdots & e_{d-3} \varphi(1,0) & e_{d-1} \varphi(1,0) & e_{d} \varphi(1,0) & \cdots & 0 \\
\ddots & \ddots & \ddots & \ddots & \ddots & \vdots \\
\cdots & 0 & 0 & 0 & \cdots & e_{1} \varphi(1,0)
\end{array}\right),
\end{aligned}
$$

and the sub-matrix $M_{2}=\left[m_{i j}^{2}\right]_{\operatorname{dim}\left(\Pi_{n-1}\right) \times \operatorname{dim}\left(\Pi_{n}\right)}$ has its entrances given by:

$$
M_{2}=\left(\begin{array}{cccccccc}
0 & 0 & 0 & \cdots & 0 & 0 & \cdots & 0 \\
\vdots & \vdots & \vdots & & \vdots & \vdots & & \vdots \\
0 & 0 & 0 & \cdots & 0 & 0 & \cdots & 0 \\
e_{2} \varphi(n, n-1) & e_{3} \varphi(1,0) & e_{4} \varphi(1,0) & \cdots & e_{d} \varphi(1,0) & 0 & \cdots & 0 \\
0 & e_{2} \varphi(n-1, n-2) & e_{3} \varphi(2,1) & \cdots & e_{d-1} \varphi(1,0) & e_{d} \varphi(1,0) & \cdots & 0 \\
\vdots & \vdots & \ddots & \ddots & \ddots & \ddots & \ddots & \vdots \\
0 & 0 & 0 & 0 & 0 & e_{2} \varphi(1,0) & \cdots & e_{d} \varphi(n, n-1)
\end{array}\right) .
$$

For the resolution of system (27) we implement the following algorithm to obtain the coefficients. As a first step, we re-ordered the coefficients $a_{(n, 0, \cdots, 0)}, a_{(n-1,1,0, \cdots, 0)}, \cdots, a_{(0, \cdots, 0, n)}$ as $a_{1}, a_{2}, \cdots, a_{\operatorname{dim}\left(\Pi_{n}\right)}$. Second, we use the fact that $M_{1}$ is an upper triangular matrix. Let the entry $(i, i)$ of $M_{1}$ correspond to the index $\left[\left(k_{1}, k_{2}, \ldots, k_{d}\right),\left(k_{1}+1, k_{2}, \ldots, k_{d}\right)\right]$, then

$$
a_{i}=-e_{1}^{2}\left(\varphi\left(k_{1}+1, k_{1}\right)\right)^{-1}\left[\sum_{j=2}^{d} e_{j} \varphi\left(k_{j}+1, k_{j}\right) a_{\left(k_{1}, \ldots, k_{j}+1, \ldots, k_{d}\right)}\right],
$$

where

$$
a_{i} \leftrightarrow M_{(i, i)} \leftrightarrow M_{\left(k_{1}, k_{2}, \ldots, k_{d}\right),\left(k_{1}+1, k_{2}, \ldots, k_{d}\right)}
$$

For the implementation of the algorithm we use the following matrix representation:

$$
\begin{gathered}
E_{1}=\left(\begin{array}{lll}
0 & 1 & 0 \\
0 & 0 & 1 \\
1 & 0 & 0
\end{array}\right), \quad E_{2}=\left(\begin{array}{ccc}
0 & \omega & 0 \\
0 & 0 & \omega^{2} \\
1 & 0 & 0
\end{array}\right), \quad E_{3}=\left(\begin{array}{ccc}
1 & 0 & 0 \\
0 & \omega & 0 \\
0 & 0 & \omega^{2}
\end{array}\right) . \\
E_{1}^{2}=\left(\begin{array}{lll}
0 & 0 & 1 \\
1 & 0 & 0 \\
0 & 1 & 0
\end{array}\right), \\
E_{1} E_{2}=\left(\begin{array}{ccc}
0 & 0 & 1 \\
\omega^{2} & 0 & 0 \\
0 & \omega & 0
\end{array}\right), \\
\left.\begin{array}{ccc}
1 & 0 & 0 \\
0 & \omega & 0
\end{array}\right), \quad \omega_{3}^{2}=\left(\begin{array}{ccc}
1 & 0 & 0 \\
0 & \omega^{2} & 0 \\
0 & 0 & \omega
\end{array}\right),
\end{gathered}
$$

This representation determines a sub-algebra of $\mathcal{C}_{3}^{1 / 3}$, yielding the extra condition $E_{1} E_{3}=E_{2}$.

Example 3.9 To illustrate the structure of $M$ and $A$, consider the case of $d=3$ and the Mittag-Leffer function $E_{\frac{2}{3}, 1}(z)=\sum_{k=0}^{\infty} \frac{z^{k}}{\Gamma(1+2 k / 3)}$. We recall that $\varphi(a, b)=\frac{\Gamma\left(1+\frac{2 a}{3}\right)}{\Gamma\left(1+\frac{2 b}{3}\right)}$ (see Example 2.2). Taking into account Corollary 3.8, the vector $A$ and the matrices $M_{1}, M_{2}$ take the form

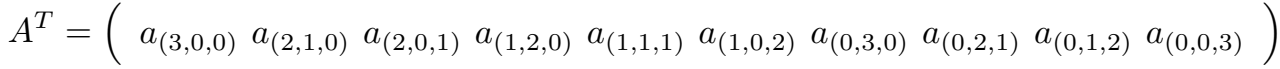

$$
\begin{aligned}
& =\left(\begin{array}{llllllllll}
a_{1} & a_{2} & a_{3} & a_{4} & a_{5} & a_{6} & a_{7} & a_{8} & a_{9} & a_{10}
\end{array}\right)
\end{aligned}
$$




$$
\begin{gathered}
M_{1}=\left(\begin{array}{cccccc}
E_{1} \varphi(3,2) & E_{2} \varphi(1,0) & E_{3} \varphi(1,0) & 0 & 0 & 0 \\
0 & E_{1} \varphi(2,1) & 0 & E_{2} \varphi(2,1) & E_{3} \varphi(1,0) & 0 \\
0 & 0 & E_{1} \varphi(2,1) & 0 & E_{2} \varphi(1,0) & E_{3} \varphi(2,1) \\
0 & 0 & 0 & E_{1} \varphi(1,0) & 0 & 0 \\
0 & 0 & 0 & 0 & E_{1} \varphi(1,0) & 0 \\
0 & 0 & 0 & 0 & 0 & E_{1} \varphi(1,0)
\end{array}\right) \\
M_{2}=\left(\begin{array}{cccc}
0 & 0 & 0 & 0 \\
0 & 0 & 0 & 0 \\
0 & 0 & 0 & 0 \\
E_{2} \varphi(3,2) & E_{3} \varphi(1,0) & 0 & 0 \\
0 & E_{2} \varphi(2,1) & E_{3} \varphi(2,1) & 0 \\
0 & 0 & E_{2} \varphi(1,0) & E_{3} \varphi(3,2)
\end{array}\right) .
\end{gathered}
$$

Now we can see that the columns of the matrix $M_{2}$ are associated, respectively, to the last four elements of the matrix A. Therefore, if we fix $a_{7}, a_{8}, a_{9}, a_{10}$ we can obtain, via formula (28), the remaining elements of the matrix $A$ as follows:

$$
\begin{aligned}
& a_{1}=-E_{1}^{2}(\varphi(3,2))^{-1}\left[\begin{array}{lll}
E_{2} \varphi(1,0) & a_{(2,1,0)}+E_{3} \varphi(1,0) & a_{(2,0,1)}
\end{array}\right], \\
& a_{2}=-E_{1}^{2}(\varphi(2,1))^{-1}\left[\begin{array}{lll}
E_{2} \varphi(2,1) & a_{(1,2,0)}+E_{3} \varphi(1,0) & a_{(1,1,1)}
\end{array}\right], \\
& a_{3}=-E_{1}^{2}(\varphi(2,1))^{-1}\left[\begin{array}{lll}
E_{2} \varphi(1,0) & a_{(1,1,1)}+E_{3} \varphi(2,1) & a_{(1,0,2)}
\end{array}\right], \\
& a_{4}=-E_{1}^{2}(\varphi(1,0))^{-1}\left[\begin{array}{lll}
E_{2} \varphi(3,2) & a_{(0,3,0)}+E_{3} \varphi(1,0) & a_{(0,2,1)}
\end{array}\right], \\
& a_{5}=-E_{1}^{2}(\varphi(1,0))^{-1}\left[\begin{array}{lll}
E_{2} \varphi(2,1) & a_{(0,2,1)}+E_{3} \varphi(2,1) & a_{(0,1,2)}
\end{array}\right], \\
& a_{6}=-E_{1}^{2}(\varphi(1,0))^{-1}\left[\begin{array}{lll}
E_{2} \varphi(1,0) & a_{(0,1,2)}+E_{3} \varphi(3,2) & a_{(0,0,3)}
\end{array}\right],
\end{aligned}
$$

which concludes the solution to the system (27). Furthermore, we can use the previous conclusions to obtain the four polynomials which are the basis for the space of fractional homogeneous monogenic polynomials $\mathcal{M}_{3}$

$$
\begin{aligned}
V_{1}^{3, \frac{2}{3}}(x) & =-x_{1}^{3}+\frac{27 \sqrt{3}}{8 \pi} E_{3}^{2} x_{1}^{2} x_{2}-\frac{27 \sqrt{3}}{8 \pi} E_{3} x_{1} x_{2}^{2}+x_{2}^{3}, \\
V_{2}^{3, \frac{2}{3}}(x) & =-E_{2}^{2} x_{1}^{2} x_{2}+E_{3}^{2} x_{1}^{2} x_{3}-E_{1} E_{2} x_{1} x_{2}^{2}-\frac{\Gamma\left(\frac{7}{3}\right)}{\Gamma^{2}\left(\frac{5}{3}\right)} E_{3} x_{1} x_{2} x_{3}+x_{2}^{2} x_{3}, \\
V_{3}^{3, \frac{2}{3}}(x) & =\omega E_{2} E_{3} x_{1}^{2} x_{2}-E_{2}^{2} x_{1}^{2} x_{3}-\frac{\Gamma\left(\frac{7}{3}\right)}{\Gamma^{2}\left(\frac{5}{3}\right)} E_{1}^{2} E_{3} x_{1} x_{2} x_{3}-E_{3} x_{1} x_{3}^{2}+x_{2} x_{3}, \\
V_{4}^{3, \frac{2}{3}}(x) & =-x_{1}^{3}+\frac{27 \sqrt{3}}{8 \pi} \omega E_{2} E_{3} x_{1}^{2} x_{3}-\frac{27 \sqrt{3}}{8 \pi} E_{1} E_{2} x_{1} x_{3}^{2}+x_{3}^{3} .
\end{aligned}
$$

For the convenience of the reader we will also write the basic monogenic polynomials for $\mathcal{M}_{1}$ and $\mathcal{M}_{2}$, respectively, as follows:

$$
\begin{aligned}
V_{1}^{1, \frac{2}{3}}(x) & =-E_{3} x_{1}+x_{2}, \\
V_{2}^{1, \frac{2}{3}}(x) & =-E_{1} E_{2} x_{1}+x_{3} . \\
V_{1}^{2, \frac{2}{3}}(x) & =E_{3}^{2} x_{1}^{2}-\frac{\Gamma\left(\frac{7}{3}\right)}{\Gamma^{2}\left(\frac{5}{3}\right)} E_{3} x_{1} x_{2}+x_{2}^{2}, \\
V_{2}^{2, \frac{2}{3}}(x) & =-\frac{\Gamma^{2}\left(\frac{5}{3}\right)}{\Gamma\left(\frac{7}{3}\right)} E_{2}^{2} x_{1}^{2}-E_{1} E_{2} x_{1} x_{2}-E_{3} x_{1} x_{3}+x_{2} x_{3}, \\
V_{3}^{2, \frac{2}{3}}(x) & =\omega^{-2} E_{2} E_{3} x_{1}^{2}-\frac{\Gamma\left(\frac{7}{3}\right)}{\Gamma^{2}\left(\frac{5}{3}\right)} E_{1} E_{2} x_{1} x_{3}+x_{3}^{2} .
\end{aligned}
$$

Remark 3.10 The above algorithm can be easily implemented. For the convenience of the reader a Matlab program for the D-G-L operators (see Example 2.2) is available at 
The main program is coef_frac(I). This program calculates the coefficients of the monogenic homogeneous polynomials that form the basis of the space of fractional homogeneous monogenic polynomials $\mathcal{M}_{l}$, i.e., solves the system (27). The output is given as cells of $3 \times 3$ representing the linear combination of the elements

$$
\left\{I_{3}, E_{1}, E_{2}, E_{3}, E_{1}^{2}, E_{2}^{2}, E_{3}^{2}, E_{1} E_{2}, E_{2} E_{3}\right\}
$$

where the coefficients for each polynomial are given by each column ordered according to Multi-indices given by the function MultiindexIndexgen. The input data of this program consists of the degree of homogeneity I.

The auxilliar program coef_frac_final_form $(A\{r, c\})$ reads each cell of the output of the main program and presents the coefficients involved in the linear combination indicated previously. The input of this program is each cell of the output of the main program.

For the case presented in the previous example first we should call the main program in the form coef_frac(3) to generate all the coefficients. After that, in order to obtain the coefficients of $V_{1}$ (similarly for $V_{2}, V_{3}$ and $V_{4}$ ) we make

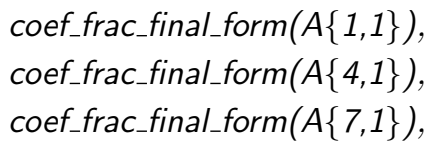

coef_frac_final_form $(A\{2,1\})$, coef_frac_final_form $(A\{5,1\})$, coef_frac_final_form $(A\{8,1\})$, coef_frac_final_form $(A\{10,1\})$. coef_frac_final_form $(A\{3,1\})$, coef_frac_final_form $(A\{6,1\})$, coef_frac_final_form $(A\{9,1\})$,

Acknowledgement: The first, second, and fourth authors were supported by Portuguese funds through the CIDMA - Center for Research and Development in Mathematics and Applications, and the Portuguese Foundation for Science and Technology ("FCT-Fundação para a Ciência e a Tecnologia"), within project UID/MAT/ 0416/2013. N. Vieira was also supported by FCT Researcher Program 2014 (Ref: IF/00271/2014).

Many wishes for health and happiness to Professor Sommen on his 60th birthday, as well as thanks and appreciation for his research and methods that shed so much light on Clifford Analysis in general and inspired this paper in particular.

\section{References}

[1] F. Brackx, R. Delanghe and F. Sommen, Clifford analysis, Research Notes in Mathematics-Vol.76, Pitman Advanced Publishing, Boston - London - Melbourne, 1982.

[2] R. Delanghe, F. Sommen and V. Souček, Clifford algebras and spinor-valued functions. A function theory for the Dirac operator, Mathematics and its Applications-Vol.53, Kluwer Academic Publishers, Dordrecht etc., 1992.

[3] H. De Bie, P. Somberg and V. Souček, The metaplectic Howe duality and polynomial solutions for the symplectic Dirac operator, J. Geom. Phys., 75, (2014), 120-128.

[4] I.H. Dimovski and V.S. Kiryakova, Convolution and differential property of Borel-Dzhrbashyan transformation, Complex Analysis and Applications, Proc. Int. Conf., Varna/Bulg, 1981. (1984), 148-156.

[5] I.H. Dimovski and V.S. Kiryakova, Convolution and commutant of Gelfond-Leontiev operator of integration, Constructive function theory, Proc. Int. Conf., Varna/Bulg, 1981. (1983), 288-294.

[6] M. Ferreira and N. Vieira, Eigenfunctions and Fundamental Solutions of the Fractional Laplace and Dirac Operators: The Riemann-Liouville Case, Complex Anal. Oper. Theory, 10 (5), (2016), 1081-1100.

[7] N. Fleury and M. Rausch de Traubenberg, Finite Dimensional Representations of Clifford Algebras of Polynomials Strasbourg, CRN-PHTH/91-07 (1991)

[8] A.O. Gel'fond and A.F. Leontév, Über eine verallgemeinerung der Fourierreihe, Mat. Sb. N. Ser., 29-No.71, (1951), 477-500.

[9] K. Gürlebeck and W. Sprößig, Quaternionic and Clifford calculus for physicists and engineers, Mathematical Methods in Practice, Wiley, Chichester, 1997.

[10] R. Herrmann, Fractional Calculus - An introduction for physicists, World Scientific, 2011.

[11] R. Jagannathan On generalized Clifford algebras and their physical applications in "The Legacy of Alladi Ramakrishnan in the Mathematical Sciences", pp.465-489 
[12] U. Kähler and N. Vieira, Fractional Clifford analysis, Hypercomplex Analysis: New perspectives and applications, Trends in Mathematics, S. Bernstein, U. Kähler, I. Sabadini and F. Sommen (eds.), Birkhäuser, Basel, (2014), 191-201.

[13] V.S. Kiryakova, Multiple Dzrbashjan-Gelfond-Leontiev fractional differintegrals, International workshop on the recent advances in applied mathematics, RAAM96, State of Kuwait, Kuwait, May 4-7, 1996. A. Hamoui et al (ed.), Department of Mathematics and Computer Sciences, Kuwait University, Kuwait, (1996), 281-294.

[14] V.S. Kiryakova, Generalized fractional calculus and applications, Pitman Reserach Notes in Mathematics SeriesVol.301, Longman Scientific \& Technical, Harlow, John Wiley \& Sons, New York, 1994.

[15] O.M. Mulyava and M. M. Sheremeta, On belonging of the Gelfond-Leontiev derivative to a convergence class (in Ukrainian), Nauk. Visn. Chernivets' kogo Univ., Mat. 485, (2009), 71-77.

[16] I.N. Sneddon, The use in mathematical physics of Erdelyi-Kober operators and some of their generalizations, Fractional Calc. Appl., Proc. Int. Conf. New Haven 1974, Lectures Notes Math.-Vol.457, (1975), 37-79.

[17] M. Rausch de Traubenberg, Clifford Algebras of Polynomials, Generalized Grassman Algebras, and q-Deformed Heisenberg Algebras, Adv. Appl. Clifford Algebr., 4- No2, (1994), 131-144

[18] N. Vieira, Fischer Decomposition and Cauchy-Kovalevskaya extension in fractional Clifford analysis: the RiemannLiouville case, Proc. Edinb. Math. Soc., II. Ser., 60-No.1, (2017), 251-272. 